\title{
Universiteit
}

Leiden

The Netherlands

\section{Accuracy of Thyroid Cartilage Fenestration during Montgomery Medialization Thyroplasty}

Desuter, G.; Cartiaux, O.; Pierard, J.; Henrard, S.; Lith-Bijl, J. van; Benthem, P.P. van; Sjogren, E.

\section{Citation}

Desuter, G., Cartiaux, O., Pierard, J., Henrard, S., Lith-Bijl, J. van, Benthem, P. P. van, \& Sjogren, E. (2019). Accuracy of Thyroid Cartilage Fenestration during Montgomery Medialization Thyroplasty. Journal Of Voice. doi:10.1016/j.jvoice.2019.01.005

Version: $\quad$ Not Applicable (or Unknown)

License: $\quad$ Leiden University Non-exclusive license

Downloaded from: https://hdl.handle.net/1887/119526

Note: To cite this publication please use the final published version (if applicable). 


\title{
Accuracy of Thyroid Cartilage Fenestration During Montgomery Medialization Thyroplasty
}

\author{
*`†Gauthier Desuter, $¥$ Olivier Cartiaux, $¥$ Jonathan Pierard, §Séverine Henrard, *'I|Julie van Lith-Bijl, \\ †Peter Paul van Benthem, and tElisabeth Sjögren, ${ }^{*} \ddagger \S$ Brussels, Belgium, and $\dagger$ Leiden, and $\|$ Almere, The Netherlands
}

\begin{abstract}
Introduction. Accuracy of thyroid cartilage fenestration during Montgomery thyroplasty (MTIS) is considered a key success factor.

The primary aim of the study was to retrospectively evaluate the accuracy of fenestration.

Furthermore, recent publications indicate a possible discrepancy in MTIS voice outcomes related to gender.

The secondary aim of the study was to investigate whether the fenestration accuracy could explain this discrepancy.

Material and Method. Study was performed by virtually drawing the fenestration on a 3D CT scan as proposed by the MTIS's instructions for use (the "expected window" (EW)), and comparing it to the actually realized fenestration (the "realized window "(RW)). Four position variables, (a) surface overlap (\%), (b) the distances between RW and EW centers (mm), (c) the angle between RW and EW $\left({ }^{\circ}\right)$, and (d) the orientation of RW's center, were studied and compared to MPT (seconds) and VHI-30 scores outcomes.

A descriptive statistical analysis and comparison between males and females were performed using a Mann-Whitney $U$ test. Linear regression and multivariate analysis were also performed.

Results. The median overlapping surface was $58.8 \%$ [34.6; 75.4]. The median radius was $3.2 \mathrm{~mm}[1.7 ; 4.1]$. The median angle was $16^{\circ}[6.8 ; 21.2]$.

Results show no significant differences of overlapping surface percentage, distance, or angle by gender. Data show no correlation between voice outcome and percentage overlap, distance, or angle. However, data show better outcomes when fenestration was located in the infero-anterior orientation. All patients of this orientation were males. Conclusions. Data provided by this study advocate a maximal infero-anterior positioning of the window during MTIS. This position is more difficult to obtain in female patients.

Key Words: Surgical Accuracy-Medialization Thyroplasty-Unilateral Vocal Fold Paralysis-Outcome-Voice -Montgomery Implant.
\end{abstract}

\section{INTRODUCTION}

Unilateral vocal fold paralysis (UVFP) can lead to breathy voice, voice fatigue, dysphagia, and phonatory dyspnea. ${ }^{1-3}$ Medialization thyroplasty represents a well-established treatment option for UVFP in cases for which a permanent treatment solution is sought. It consists of restoring a glottic closure by pushing the immobile vocal fold medially by means of a material introduced into the para-glottic muscular space through a thyroid cartilage fenestration.

The Montgomery Implant System Thyroplasty (MTIS) represents a type of medialization thyroplasty that consists of a step-by-step operative procedure using a premolded hard silicone implant, which is preoperatively selected from a selection of six sizes of implants per gender. ${ }^{4-8}$

\footnotetext{
Accepted for publication January 3, 2019.

From the *Otolaryngology, Head and Neck Surgery Department, Voice and Swallowing Clinic, Cliniques Universitaires Saint-Luc, Université catholique de Louvain, Brussels, Belgium; †Otolaryngology, Head and Neck Surgery Department, Leidse Universitaire Medisch Centrum (LUMC), University of Leiden, Leiden, The Netherlands; †Neuromusculoskeletal Lab (NMSK), Institut de Recherche Clinique et Expérimentale (IREC), Université catholique de Louvain, Brussels, Belgium; §Clinical Pharmacy Research Group, Louvain Drug Research Institute, and Institute of Health and Society (IRSS), Université catholique de Louvain, Brussels, Belgium; and the $\|$ Otolaryngology Deparment., Flevoziekenhuis, Almere, The Netherlands.

Address correspondence and reprint requests to Gauthier Desuter, Voice and Swallowing Clinic, Otolaryngology Head and Neck Surgery Department, Suite 407, 10 Avenue Hipocrate, Brussels 1200, Belgium. E-mail addresses:

gauthier.desuter@uclouvain.be,gdesuter@post.harvard.edu

Journal of Voice, Vol. $\mathbf{\square}$, No. $\mathbf{\square}$, pp. $\mathbf{\square}-\mathbf{\square}$

0892-1997

(C) 2019 The Voice Foundation. Published by Elsevier Inc. All rights reserved

https://doi.org/10.1016/j.jvoice.2019.01.005
}

Essentially the cartilage window, which has a fixed size for males and for females, is placed at a fixed distance from the lower border of the thyroid cartilage and a fixed distance from the anterior midline

The MTIS is reputed to have short learning curve and long-lasting benefits in terms of Voice Handicap Index (VHI-30). ${ }^{9,10}$ However, some concerns regarding the results of female implants in terms of maximum phonation time (MPT) were recently brought up. ${ }^{11}$ Factors that could be of influence in this discrepancy in results are (a) the shape of the thyroid cartilage, (b) the shape and dimensions of the implant itself, and (c) the site at which the implant is placed, that is, the location of the cartilage fenestration. This study will investigate the last.

The primary aim of the study was to retrospectively evaluate the accuracy of fenestration taking as standard-reference the positioning proposed by MTIS instructions for use.

The secondary aim of study was to investigate whether the factor of fenestration accuracy could explain discrepancy in results by gender.

\section{MATERIAL AND METHOD}

This study was approved by the Institution Review Board under the reference $2017 / 12 \mathrm{M} / 266$.

Studied variables were two-fold: (a) position variables, and (b) outcomes variables. 

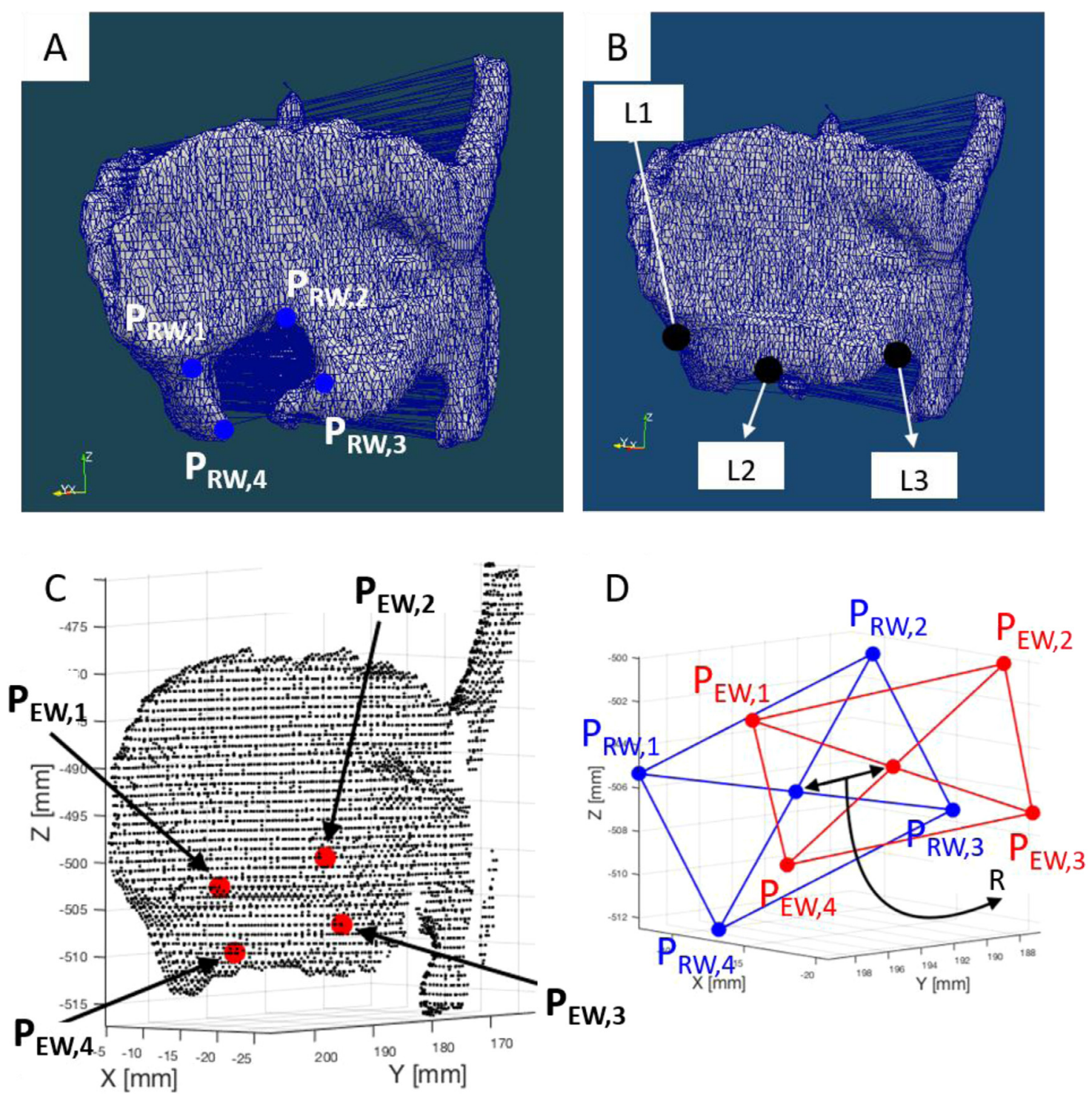

FIGURE 1. Illustrations showing A. the thyroid cartilage with the realized fenestration-blue points $\left(P_{R W, 1}, P_{R W, 2}, P_{R W, 3}, P_{R W, 4}\right)$ are the four corners of RW, B. the simulated intact thyroid cartilage and the three anatomical landmarks (L1, L2, L3) identified manually, C. the simulated intact thyroid cartilage and the computed expected fenestration-red points $\left(\mathrm{P}_{\mathrm{EW}, 1}, \mathrm{P}_{\mathrm{EW}, 2}, \mathrm{P}_{\mathrm{EW}, 3}, \mathrm{P}_{\mathrm{EW}, 4}\right)$ are the four corners of EW, and D. example of comparison between EW in red and RW in blue-R is the distance between EW and RW centers. (For interpretation of the references to color in this figure legend, the reader is referred to the Web version of this article.)

The position variables were: (a) the percentage of overlapping surfaces (PO in \%) between expected window (EW) and realized window $(\mathrm{RW})$; (b) the distance between the center of EW and RW or radius (in $\mathrm{mm}$ ), (c) the angle between EW and RW (angle), and (d) the position of RW center respective to the EW center (Quadrants [Q]) called supero-anterior $\mathrm{Q}$, supero-posterior $\mathrm{Q}$, infero-anterior $\mathrm{Q}$, and infero-posterior $\mathrm{Q}$ ).

The outcome variables were: (a) absolute (in seconds) and relative (in \%) preoperative postoperative differences in MPT and (b) absolute (n/120) and relative preoperativepostoperative differences (in \%) in VHI-30. The postoperative MPT and VHI-30 assessments took place one-month postoperative as a standard procedure.

\section{Measurement of accuracy of fenestration}

The postoperative CT scans of 28 patients (16 males and 12 females) of MTIS as sole treatment for UVFP, as defined by the European Laryngological Society guidelines, were collected. ${ }^{12}$

A postoperative 3D CT model of the thyroid cartilage with the realized fenestration, also called the realized window (RW), was segmented and reconstructed using ITKSnap; an interactive open-source software that allows 3D medical image navigation and delineation of anatomical structures. ${ }^{13} \mathrm{RW}$ was defined by manually identifying the four corners in the postoperative 3D CT model (Figure 1A). In practice, RW of each patient was recorded by storing the coordinates of the four corners of the realized fenestration 
stated in the reference frame of the CT images (Figure 1A). For planning purposes, in order to define the expected window (EW), a preoperative 3D model of the intact thyroid cartilage (before MTIS) was simulated by virtually filling the cartilage defect (caused by the fenestration) in the postoperative 3D CT model (Figure 1B). EW was then defined according to the instruction for use provided in the MTIS commercial documentation. ${ }^{14}$ The input of the step-by-step MTIS procedure consisted of three anatomical landmarks to be identified manually in the preoperative $3 \mathrm{D}$ model of the thyroid cartilage (Figure 1B). Then the step-by-step MTIS procedure was implemented using numerical computation software (MatLab, The MathWorks, Natick, Massachusetts) to compute automatically the output of the planning process as the EW (Figure 1C). In practice, EW of each patient was recorded by storing the coordinates of the four corners of the expected fenestration stated in the same reference frame than that of RW. As a result, both RW and EW of each patient can be compared quantitatively in terms of relative positioning and orientation for accuracy measurement purposes (Figure 1D).

The sensitivity of the step-by-step MTIS planning procedure was analyzed by applying random noise to the input of the automatic algorithm. In practice, each of the three anatomical landmarks identified manually in the preoperative 3D model of the thyroid cartilage was numerically perturbed by a random noise of maximum $2 \mathrm{~mm}$. We arbitrarily chose the $2 \mathrm{~mm}$ measure as error postulate. This measure corresponded to a surface covering approximately half of the length of the cartilage lower border between the inferior tuberculum and the respective extremities of the thyroid ala. The impact of these random errors on the definition of the expected fenestration was small. Variations in the dimensions of the computed EW were estimated to be within a $15 \%$ interval centered on the nominal geometry of EW.

\section{Statistical analysis}

A descriptive statistical analysis and comparison between males and females were performed using a Mann-Whitney $U$ test for each studied variable except for the Quadrants variable.
Linear regression models were performed to assess the relationship between several variables on the outcome variables, that is, the absolute increase of MPT and the relative decrease in VHI-30 before and after the surgery. All variables associated with a $P$ value $<0.20$ in univariate model were candidate for the multivariable model. The final multivariable model was chosen through stepwise selection using the Akaike's Information Criteria. In addition, multicolinearity was checked through variance inflation factor.

Line graphs were made comparing preoperative and postoperative VHI-30 according to their position in terms of quadrants. Likewise, line graphs were made comparing preoperative and postoperative MPT according to their position in terms of quadrants.

\section{RESULTS}

The increase of MPT and the decrease in VHI-30 were respectively of 6.6 seconds (164\% increase) and 45 points ( $77 \%$ decrease). There was a difference of results unfavorable to female for both outcome measures although only significant in terms of VHI-30 decrease.

Table 1 summarizes these descriptive statistics and comparison between males and females.

Results showed a median PO of $59 \%$ that is $60 \%$ for male and $56 \%$ for female, showing no significant difference of window overlapping surface between genders.

The radius, standing for the distance between the EW center and the RW center, showed a median of $3.2 \mathrm{~mm}$, $3.0 \mathrm{~mm}$ for males and $3.7 \mathrm{~mm}$ for females showing no significant difference between genders.

The angle between EW and RW, showed a median of $14^{\circ}$, $16^{\circ}$ for males and $7.5^{\circ}$ for females, showing a clear difference between genders, however not significant.

As displayed in Figure 2, there is no correlation between outcome (MPT and VHI-30) and three position variables: $\mathrm{PO}$, radius, and angle.

Figure 3 displays a three-dimensional representation of RW centers with respect to their EW centers, showing $X$ coordinates and $\mathrm{Y}$ coordinates along with the voice outcome groups they belong to. A color was attributed to their respective postoperative voice outcomes groups in terms of

TABLE 1.

Table Displays the Descriptive Statistics and the Comparison Between Males and Females, Topographical and Outcome Variables

\begin{tabular}{|c|c|c|c|c|}
\hline & $\begin{array}{c}\text { Total }(n=28) \\
\text { Median }[\text { P25; P75] }\end{array}$ & $\begin{array}{c}\text { Males }(n=16) \\
\text { Median [P25; P75] }\end{array}$ & $\begin{array}{l}\text { Females }(n=12) \\
\text { Median [P25; P75] }\end{array}$ & $P$ Value \\
\hline Overlaping surface (\%) & $58.8[34.6 ; 75.4]$ & $60.0[54.8 ; 81.3]$ & $56.2[32.2 ; 67.7]$ & 0.246 \\
\hline Angle $\left({ }^{\circ}\right)$ & $14.0[6.8 ; 21.2]$ & $16.0[9.5 ; 21.2]$ & $7.5[4.0 ; 18.7]$ & 0.099 \\
\hline VHI, absolute decrease (pts) & $45.0[24.3 ; 66.8]$ & $63.0[33.3 ; 70.8]$ & $35.0[20.8 ; 44.0]$ & 0.027 \\
\hline VHI, relative decrease (\%) & $77.0[49.8 ; 88.8]$ & $85.8[72.0 ; 91.4]$ & $56.8[48.7 ; 75.8]$ & 0.029 \\
\hline
\end{tabular}



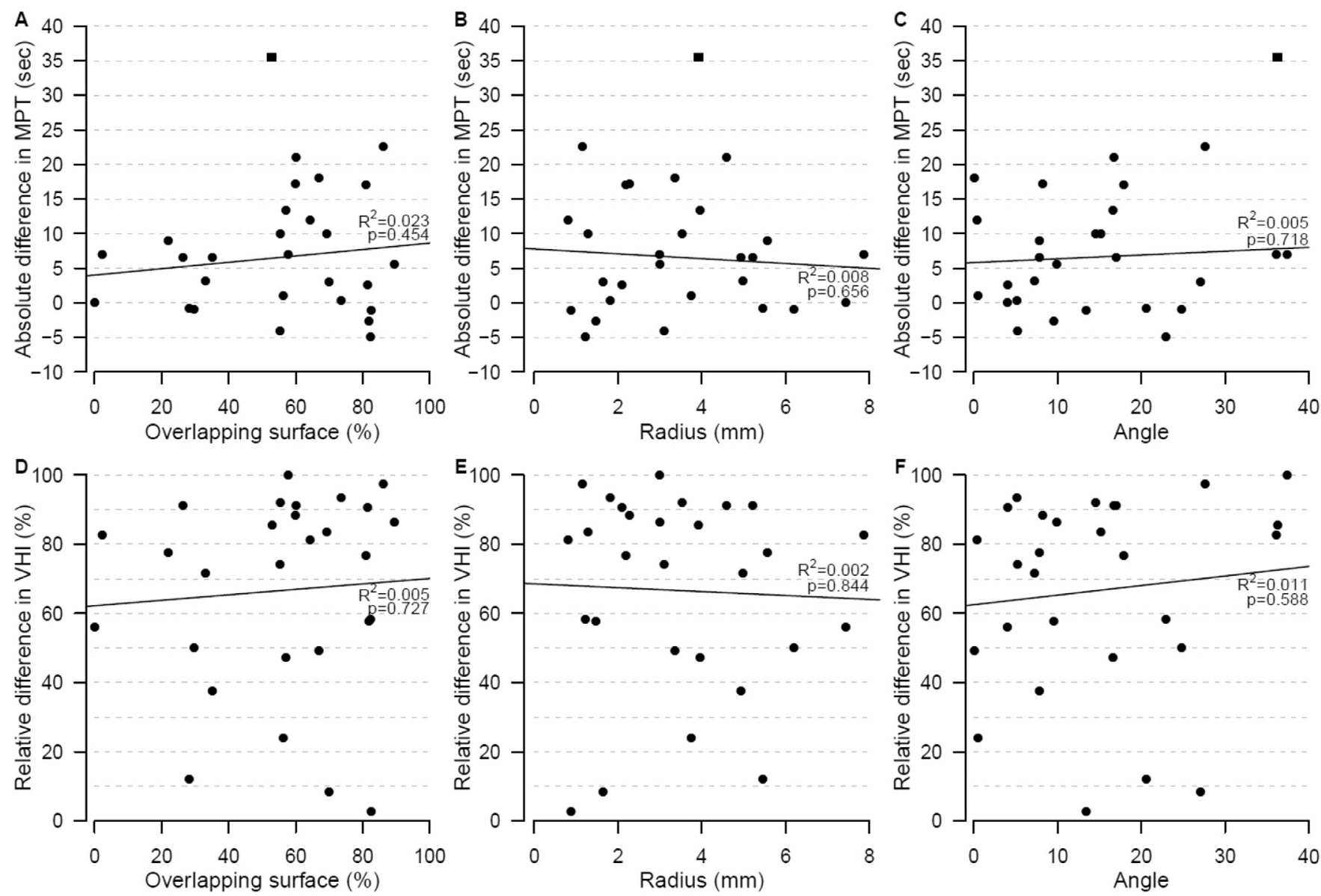

FIGURE 2. Scatter plots graphs showing A. the association between MPT and PO, B. the association between MPT and R, C. the association between MPT and angle, D. the association between VHI-30 and PO, E. the association between VHI-30 and R, F. the association between VHI-30 and angle.

relative decrease of VHI-30 and absolute increase of MPT. For this specific figure, the choice for a relative change in VHI-30 rather than an absolute one, was made to minimize subjective self-perception differences that exist between individuals.

Figure 4 displays linear graphs showing preoperative and postoperative results in terms of (A) absolute increase of MPT and (B) absolute decrease of VHI-30 by Quadrants. For this specific graphic representation of results the use of relative changes of VHI-30 was impossible. Results of Figure 4 are aligned with results of Figure 3.

Indeed, according to both Figures 3 and 4 the infero-anterior $\mathrm{Q}$ location of implants centers shows better voice outcomes. Accordingly, infero-anterior $\mathrm{Q}$ will, by postulate, be considered as the reference $\mathrm{Q}$ within the consecutive multivariate analysis.

Table 2 displays the results of the univariate and multivariable regression analysis of variables associated with the absolute increase of MPT and with the relative decrease in VHI-30.

The multivariate analysis confirms a significant correlation between a positioning of the fenestration within the infero-anterior $\mathrm{Q}$ and voice results in terms of absolute increase of MPT and relative decrease of VHI-30.

\section{DISCUSSION}

\section{Fenestration accuracy}

The $2 \mathrm{~mm}$ EW determination error postulate corresponds to the measured lowest radius variability of the surgeon $(1.8 \mathrm{~mm})$. This validates, post-hoc, the chosen error postulate.

The overall percentage of surface overlap between RW and $\mathrm{EW}$ is rather low $(59 \%)$. Likewise, the median distance separating RW centers with EW centers is rather high $(3.2 \mathrm{~mm})$. Finally, the median angle between EW and RW is also rather large $\left(14^{\circ}\right)$. These data demonstrate the presence of variability in terms of fenestration location when compared with the MTIS instructions for use provided by Bess Inc.

Nonetheless, no correlation was found between these two position variables and voice outcome variables.

Three hypotheses could explain this variability that does not affect outcome: (a) the MTIS procedure, as described by Bess Inc., does not represent a "one-fits-all" procedure but rather a guideline procedure that needs to be interpreted by surgeons, (b) the MTIS procedure does represent indeed a "must-follow" procedure that allows some variability before affecting outcome and finally, (c) surfaces of overlap, radius, and angle does not represent pertinent position variables. 


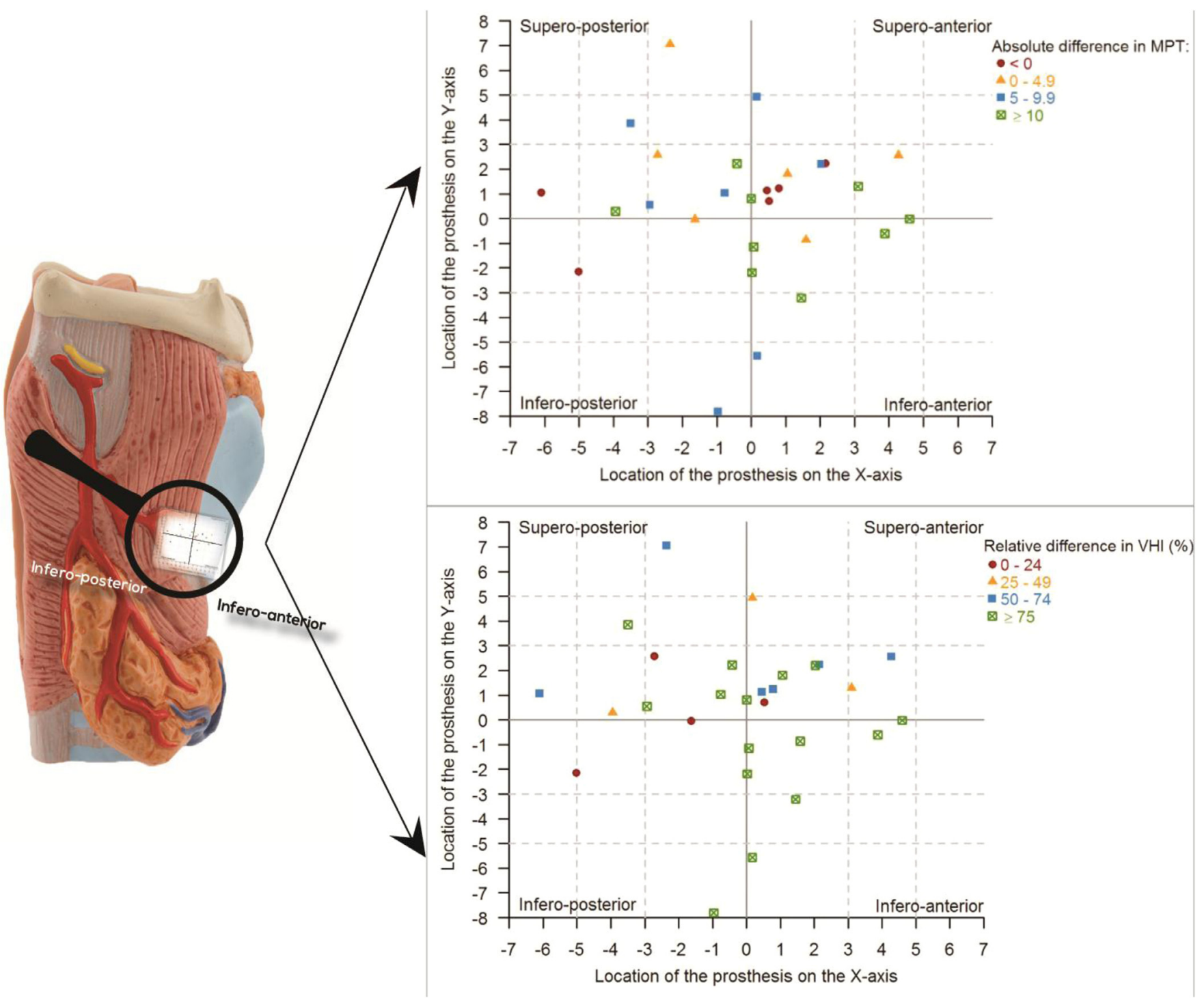

FIGURE 3. Topographical plotting of RW centers with respect to their EW centers. A color was attributed to their respective postoperative outcomes in terms of absolute increase of MPT (above) and in terms of relative decrease of VHI-30 (below). (For interpretation of the references to color in this figure legend, the reader is referred to the Web version of this article.)

The results obtained by the last position variable, the Quadrant, give some credit to this very last hypothesis. Indeed, data show evidence that patients that benefited from a more anterior and inferior fenestration positioning have better voice outcomes.

These data may confirm an MTIS surgeon's intuition that is that a most infero-anterior fenestration will avoid any protrusion of the implant within the Morgani's ventricule as well any exaggerated interaction with the arytenoid cartilage posteriorly.

These study findings are aligned with a very recent study published by C. Storck et al that focused on the impact of the MTIS implant on the arytenoid cartilage. ${ }^{15}$ Authors demonstrated that the MTIS implant causes a gentle superior and posterior push on the cricoidal facet of the arytenoid cartilage causing a medialization and a rising of the paralyzed vocal fold. Achieving this arytenoid move will require an implant positioning that is low and anterior.
Finally, while RW are equally distributed between three of the quadrants, one can observe that almost none of the $\mathrm{RW}$-independent of patient gender-were located in the infero-posterior quadrant. Authors believe that is due to the presence of the thyro-hyoid muscle that surgeons tend to approach very conservatively. No other operative pattern that the surgeon would have developed with the time could be identified.

\section{Outcome discrepancy between genders}

There is a notable, although not significant, difference of angle between EW and RW related to gender. Authors think this might be related to the respective usable area for implantation; this is the area beneath a horizontal line through the midpoint, and the anterior border of the thyrohoid muscle. This area is smaller in females than in males, allowing less angle variability. 

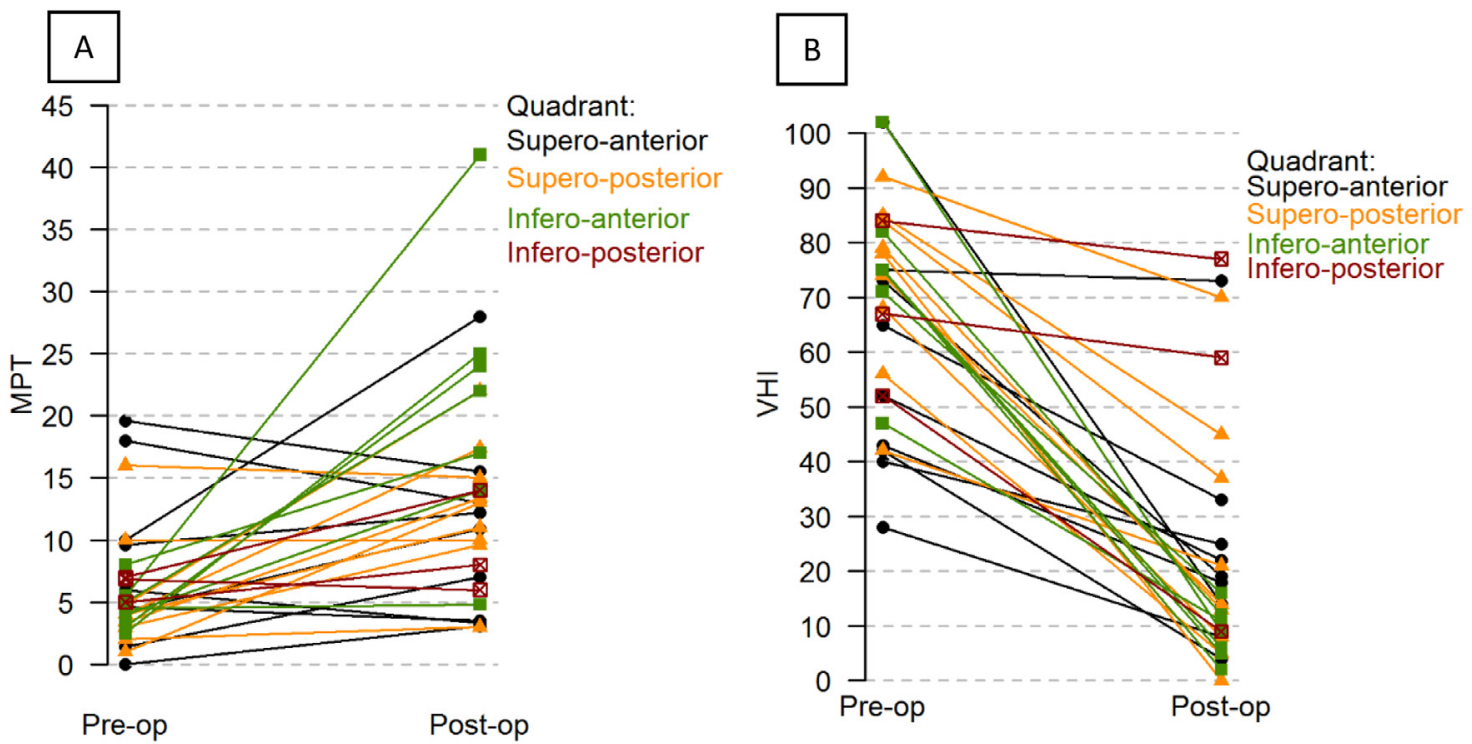

FIGURE 4. Linear graphs showing preoperative and postoperative differences by Quadrant (Q), in terms of A. absolute increase of MPT and $\mathbf{B}$. in terms of absolute decrease of VHI-30.

\section{TABLE 2.}

Univariate and Multivariate Regression Analysis of Variables Associated With A. the Absolute Increase of MPT and B. the Relative Decrease of VHI-30. All Variables Associated with a $P$ Value of $<0.2$ in Univariate Model were Candidate for the Multivariable Model

A. Variables Associated With the Absolute Increase of MPT (s)

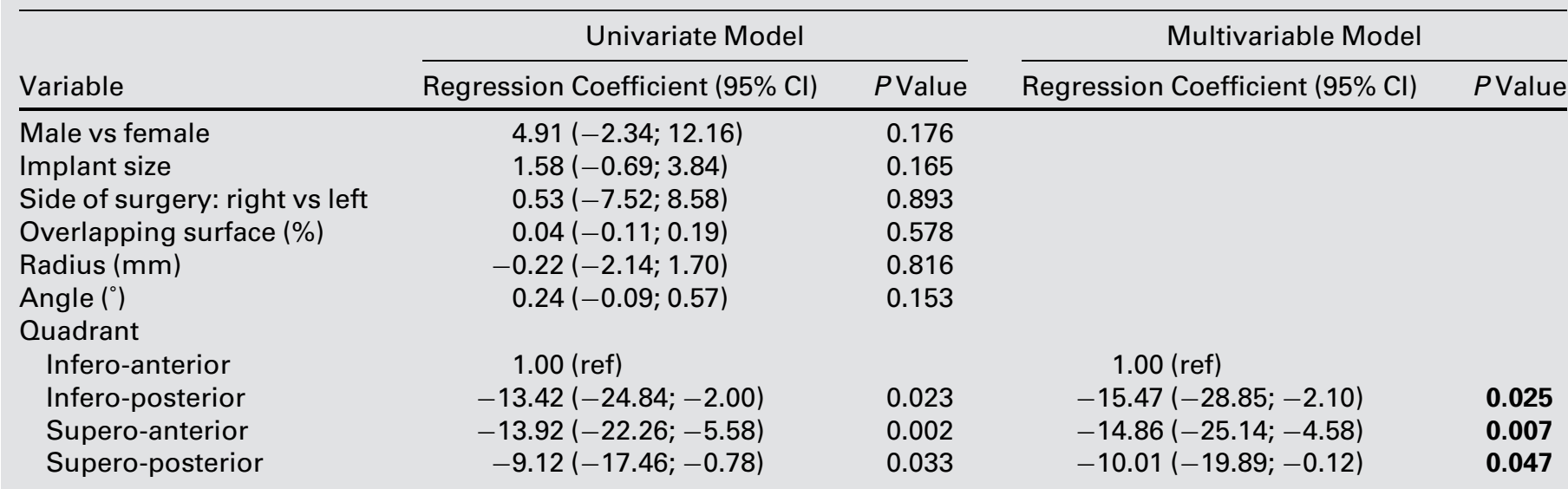

B. Variables Associated With the Relative Decrease of VHI-30 (\%)

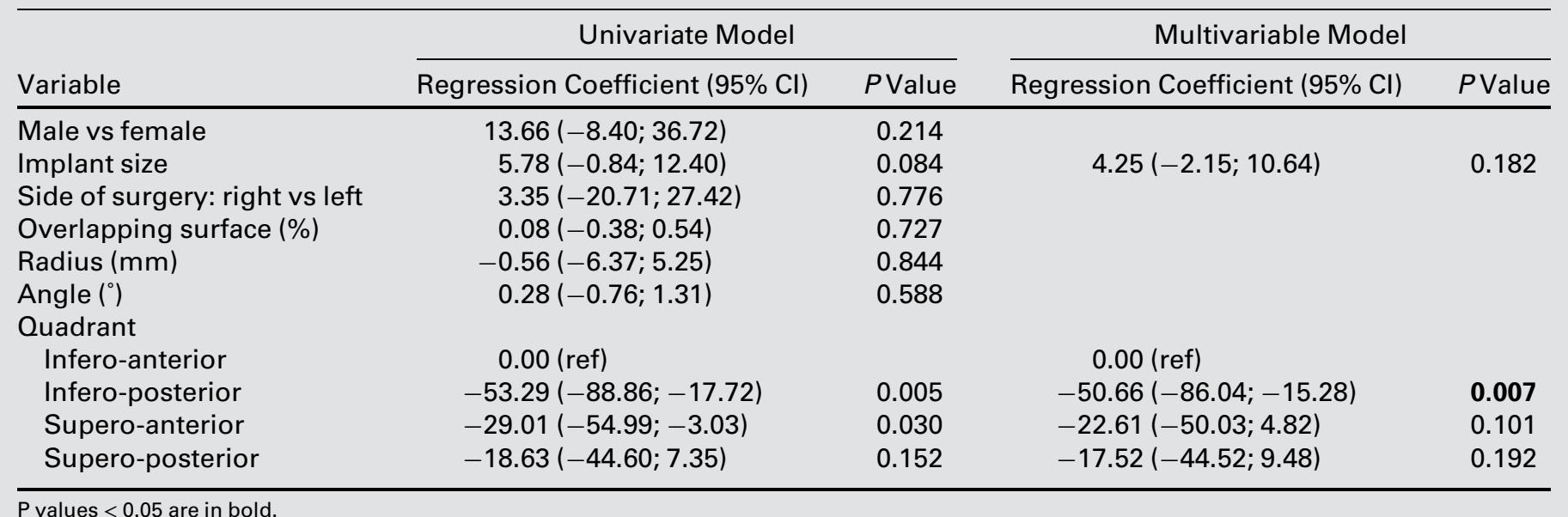

$P$ values $<0.05$ are in bold. 
Furthermore, this study shows that none of cohort's patients that had their RW centers in the infero-anterior quadrant were female.

This could represent a factor affecting MTIS outcome in female individuals.

Two hypotheses can be formulated to explain this gender discrepancy: (a) the MTIS instruction for use are simply wrong for female individuals or (b) size and/or shape of the stabilizing mid-plate of the MTIS implant does not allow a maximal infero-anterior positioning of the fenestration.

Finally, some limitations of the study have to be underscored.

Window location does certainly not represent the sole factor influencing voice outcome. Factors such as volume of the inner part of the prosthesis, induced tissue compression, ${ }^{16}$ and eventually shape of the implant that possibly influences ${ }^{17-19}$ the arytenoid cartilage position, represents other outcome factors to consider.

The population of the study is rather limited and CT images were collected retrospectively with some variability in image acquisition parameters. Furthermore, EW location was defined by one sole engineer, potentially inducing a single-evaluator bias. Nonetheless, the sensitivity analysis performed by applying the $2 \mathrm{~mm}$ random noise on the input of the numerical MTIS planning process has shown a small impact on the definition of the expected fenestration.

\section{CONCLUSION}

Despite good overall outcome results, window positioning accuracy of MTIS fenestration is rather low for both genders. Therefore, the MTIS landmarks provided by Bess Inc. step-by-step procedure should, to a certain extent, not be considered as too rigid.

Data provided by this study advocate a maximal inferoanterior positioning of the window during MTIS. This maximal infero-anterior positioning is more difficult to obtain for female patients. Authors hypothesize that an improper design of female rectangle base and/or an improper female step-by-step surgical procedure impedes upon maximal infero-anterior location of the female prosthesis.

Further prospective studies should investigate the rectangle base, as well as the intra-laryngeal portion of the MTIS prosthesis as a possible determinant of voice outcome by gender, with a maximal infero-anterior fenestration position as standard.

\section{REFERENCES}

1. Mattei A, Desuter G, Roux M, et al. International consensus (ICON) on basic voice assessment for unilateral vocal fold paralysis. Eur Ann Otorhinolaryngol Head Neck Dis. 2018;135:S11-S15.
2. Bonilla-Velez J, Small M, Bonilla-Escobar FJ, et al. Voice and swallowing outcomes of unilateral vocal fold paralysis: comparing younger adult and geriatric patients. Otolaryngol Head Neck Surg. 2018;158:904-911.

3. Rosen CA, Mau T, Remacle M. Nomenclature proposal to describe vocal fold motion impairment. Eur Arch Otorhinolaryngol. 2016:273:1995-1999.

4. McLean-Muse A, Montgomery WW, Hillman RE. Montgomery thyroplasty implant for vocal fold immobility: phonatory outcomes. Ann Otol Rhinol Laryngol. 2000;109:393-400.

5. Montgomery WW, Blaugrund SM, Varvares MA. Thyroplasty: a new approach. Ann Otol Rhinol Laryngol. 1993;102:571-579.

6. Laccourreye O, Benkhatar H, Ménard M. Lack of adverse events aftermedialization laryngoplasty with the montgomery thyroplasty implant in patientswith unilateral laryngeal nerve paralysis. Ann Otol Rhinol Laryngol. 2012;121:701-707.

7. Laccourreye O, El Sharkawy L, Holsinger FC, et al. Thyroplasty type I with Montgomery implant among native French language speakers with unilateral laryngeal nerve paralysis. Laryngoscope. 2005; 115:1411-1417.

8. Nouwen J, Hans S, De Mones E. Thyroplasty type I without arytenoid adduction in patients with unilaterallaryngeal nerve paralysis: the montgomery implant versus the Gore-Tex implant. Acta Otolaryngol. 2004:124:732-738.

9. Desuter G, Henrard S, Boucquey D. Learning curve of medialization thyroplasty using a Montgomeryimplant. Eur Arch Otorhinolaryngol. 2015;272:385-390.

10. Desuter G, Zapater E, Van der Vorst S. Very long-term voice handicap index voice outcomes after Montgomery thyroplasty: a cross-sectional study. Clin Otolaryngol 2018. https://doi.org/10.1111/coa.13113. [Epub ahead of print]

11. Desuter G, Henrard S, van Lith-Bijl JT. Shape of thyroid cartilage influences outcome of montgomery medialization thyroplasty: a gender issue. $J$ Voice. 2017:31:245. e3-245.e8.

12. Rosen CA, Mau T, Remacle M, et al. Nomenclature proposal to describe vocal fold motion impairment. Eur Arch Otorhinolaryngol. 2016;273:1995-1999

13. Yushkevich PA, Piven J, Hazlett HC, et al. User-guided 3D active contour segmentation of anatomical structures: significantly improved efficiency and reliability. Neuroimage. 2006;31:1116-1128.

14. Montgomery ${ }^{\circledR}$ Thyroplasty Implant System: Instructions for Use. Boston Medical Products, Shrewbury, MA, USA; MK-THYCAT-C; 05-2016.

15. Storck C, Lüthi M, Honegger F, et al. Surgical impact of the montgomery implant system on arytenoid cartilage and the paralyzed vocal fold. $J$ Voice 2018. https://doi.org/10.1016/j.jvoice.2018.07.019. pii: S0892-1997(18)30232-7[Epub ahead of print] PubMed PMID: 30172670 .

16. Benninger MS, Chota RL, Bryson PC, et al. Custom implants for medicalization laryngoplasty: a model that considers tissue compression. $J$ Voice. 2015;29:363-369.

17. Chester MW, Stewart MG. Arytenoid adduction combined with medicalization thyroplasty: an evidence-based review. Otolaryngol Head Neck Surg. 2003;129:305-310

18. Li AJ, Johns MM, Jackson-Menaldi C, et al. Glottic closure patterns: type I thyroplasty versus type I thyroplasty with arytenoid adduction. $J$ Voice. 2011;25:259-264.

19. Chang J, Schneider SL, Curtis J, et al. Outcomes of medialization laryngoplasty with and without arytenoid adduction. Laryngoscope. 2017;127:2591-2595. 